\title{
Outdoor Ranging and Positioning based on LoRa Modulation
}

\author{
Pascal Müller, Hannes Stoll and Luciano Sarperi \\ Institute of Signal Processing and Wireless Communications \\ ZHAW School of Engineering \\ Winterthur, Switzerland \\ \{pascal.mueller, hannes.stoll, luciano.sarperi\}@zhaw.ch
}

\author{
Christof Schüpbach \\ armasuisse Science + Technology \\ Thun, Switzerland \\ christof.schuepbach@armasuisse.ch
}

\begin{abstract}
We present results for outdoor ranging and positioning based on the Long-Range (LoRa) modulation, which is a widespread wireless IoT technology. LoRa systems typically operate in sub-GHz frequency bands with only $125 \mathrm{kHz}$ bandwidth, which restricts the ranging performance due to the limited temporal resolution. The investigated LoRa ranging method operates in the $2.4 \mathrm{GHz}$ ISM frequency band and supports wider bandwidths, achieving for outdoor ranging a mean distance error below $50 \mathrm{~m}$ over distances of up to $1400 \mathrm{~m}$. Positioning based on LoRa ranging obtained a measured Distance Root Mean Square (DRMS) error of $30 \mathrm{~m}$, which reduced to $5 \mathrm{~m}$ for tracking of a mobile node, making it a suitable method for approximate outdoor positioning and tracking of IoT devices without using Global Navigation Satellite Systems (GNSS).
\end{abstract}

Keywords-Long-Range (LoRa) Modulation, SX1280, $2.4 \mathrm{GHz}$, ranging, positioning, localization, tracking

\section{INTRODUCTION}

The Long-Range (LoRa) modulation is based on Chirp Spread Spectrum (CSS) signals and has become popular for wide-area Internet of Things (IoT) applications due to the operation in unlicensed spectrum (typically $868 \mathrm{MHz}$ in Europe and $915 \mathrm{MHz}$ in North America), its energy efficiency and low cost [1]. Since IoT devices are typically energy constrained and price-sensitive, the localization of IoT devices cannot always rely on an additional Global Navigation Satellite Systems (GNSS) receiver. Another reason for not relying on GNSS can also be that the line-of-sight (LOS) path to the satellites may be blocked.

LoRa operating in the $868 \mathrm{MHz}$ frequency band typically uses $125 \mathrm{kHz}$ of bandwidth where Time-Difference-ofArrival (TDoA) methods have been shown to obtain median location errors of around $150 \mathrm{~m}$ [2]. As shown in section II.C, the temporal resolution of such a LoRa signal corresponds to a minimum separable difference in length of individual paths of $2400 \mathrm{~m}$. In oder to obtain higher temporal resolution for time-of-flight (TOF) based ranging in multipath propagation environments the SX1280 LoRa transceiver is employed, which operates in the $2.4 \mathrm{GHz}$ ISM frequency band and supports bandwidths of up to $1625 \mathrm{kHz}$. After a brief introduction to ranging with LoRa we present measurement results obtained for ranging as well as positioning and tracking in outdoor environments. The results based on practical measurements show that LoRa ranging can be used for determining approximate ranging distances or locations and for tracking of LoRa enabled IoT devices without the need for an additional GNSS receiver.

\footnotetext{
This work was supported by armasuisse Science + Technology
}

Other common IoT wireless technologies operating in the 2.4 GHz ISM frequency band are Bluetooth Low Energy (BLE) and Wi-Fi. While BLE with the direction finding functionality is well suited for short-range localization of nodes by triangulation, the coded-PHY long-range mode does not support direction finding [3]. Several localization methods using Wi-Fi exist, but its limited range precludes wide-area applications.

Previous work on LoRa ranging in [4] provides a detailed performance comparison between LoRa ranging and Coherent Ranging On Narrowband Enabled Networks (CRONEN). LoRa ranging in the $2.4 \mathrm{GHz}$ frequency band obtained a mean error of $75 \mathrm{~m}$ for LOS and $144 \mathrm{~m}$ for non-LOS environments for distances $\leqslant 500 \mathrm{~m}$, while the mean error values were around $50 \%$ lower with CRONEN. The investigation of the LoRa ranging capabilities in [5] presents additional ranging measurement results for different LoRa parameter settings. Compared to [4] and [5], our main contribution is that we extend the use of LoRa ranging to positioning and tracking.

\section{RANGING WITH LORA MODULATION}

\section{A. Basics of LoRa Ranging}

LoRa uses $2^{S F}$ possible cyclic time shifts of a CSS symbol for modulation, with one symbol carrying $S F$ bits. The value $S F$ is termed spreading factor, although its role is different from the one in traditional Code Division Multiple Access (CDMA) systems since the bandwidth $B W$ can be chosen independently of the spreading factor [6]. The SX1280 transceiver operates in the $2.4 \mathrm{GHz}$ ISM frequency band, where larger bandwidths are available than in the lower frequency bands, supporting the bandwidths $B W=406,812$ and $1625 \mathrm{kHz}$ and spreading factors $S F=5,6, \ldots, 10$ for ranging. The highest RX sensitivity is $-122 \mathrm{dBm}$, which is achieved with $B W=406 \mathrm{kHz}$ and $S F=10$ [7] and results in a maximum coupling loss of $134.5 \mathrm{~dB}$ when the TX power at its maximum value of $12.5 \mathrm{dBm}$. This corresponds up to a few kilometers coverage in a rural environment.

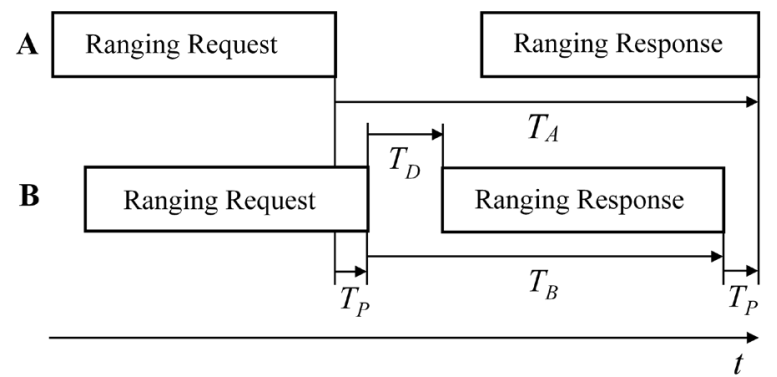

Fig. 1. Two-way ranging principle with LoRa ranging in the SX1280 transceiver 
The ranging engine in the SX1280 PHY layer uses a twoway ranging procedure for half-duplex systems described in section 6.1 of [8], which is based on TOF measurements and shown in Fig. 1, with ranging reference time-points defined at the end of the ranging messages. Compared to one-wayranging methods, the clocks in node $\mathrm{A}$ and node $\mathrm{B}$ do not have to be time synchronized, but clock oscillator offsets between the two nodes should be kept small.

Upon reception of a ranging request message from node $\mathrm{A}$ containing the correct node $\mathrm{B}$ address, node $\mathrm{B}$ switches $T_{D}=2 \cdot T_{\text {sym }}$ seconds after the end of the ranging request from receive to transmit mode and sends a ranging response message to node A. The switching delay $T_{D}$ depends on the CSS symbol duration $T_{\text {sym }}=2^{S F} / B W$.

By measuring the time $T_{A}$, which is assumed to have been corrected for known delays additional to the propagation overthe-air and which are determined during calibration, the propagation time $T_{P}$ and the ranging distance $d$ can be obtained by node $\mathrm{A}$ as

$$
d=c \cdot T_{P}=c \cdot \frac{T_{A}-T_{B}}{2}
$$

where $c$ is the propagation speed and $T_{B}$ is the overall response delay between ranging request reception and ranging response transmission at node $\mathrm{B}$, which includes the switching delay $T_{D}$ and the duration of the ranging response configured to $15 \cdot T_{\text {sym }}$.

In section 6.1.3. of [8] it is shown that in such a system the error of the measured propagation time mainly depends on the relative oscillator offset $\delta=\left(f_{B}-f_{A}\right) / f_{0}$ between the clockfrequency of the node $\mathrm{B} f_{B}$ and node $\mathrm{A} f_{A}$, with $f_{0}$ being the nominal frequency. This results in the approximate ranging distance error

$$
\Delta d \approx-c \cdot \frac{\delta \cdot T_{B}}{2}
$$

Assuming $\delta= \pm 40$ ppm (unlikely worst-case with the accuracy of the node A and node B clock each being \pm 20 $\mathrm{ppm}$ ), this results for the longest response delay $T_{B}=42.9 \mathrm{~ms}$ obtained according to [7] for $B W=406 \mathrm{kHz}$ and $S F=10$ in a theoretical worst-case ranging distance error of $\Delta d_{\max }=\mp$ $257 \mathrm{~m}$. With the parameters $B W=1625 \mathrm{kHz}$ and $S F=5$ the error is minimised and reduces to $\Delta d_{\min }=\mp 2.01 \mathrm{~m}$. Therefore, for long CSS symbol durations $T_{\text {sym }}$ a correction of the relative clock offsets will be more beneficial.

The relative oscillator offset $\delta$ can be obtained by measuring the carrier frequency offset, which however is only possible when transmitting standard (non-ranging) LoRa messages with the SX1280 transceiver. Instead, a back-toback ranging was implemented to minimise the impact of the relative oscillator offset, as described subsequently.

\section{B. Implementation of LoRa Ranging}

The implemented back-to-back ranging uses two two-way ranging procedures, where the roles of node $\mathrm{A}$ and node $\mathrm{B}$ are swapped during the second two-way ranging procedure according to section 6.1.3 in [8] and which is summarised in the message flow diagram in Fig. 2. Since the ranging distance errors $\Delta d$ in (2) will have the same magnitude but opposite signs for the respective ranging procedures, the error due to

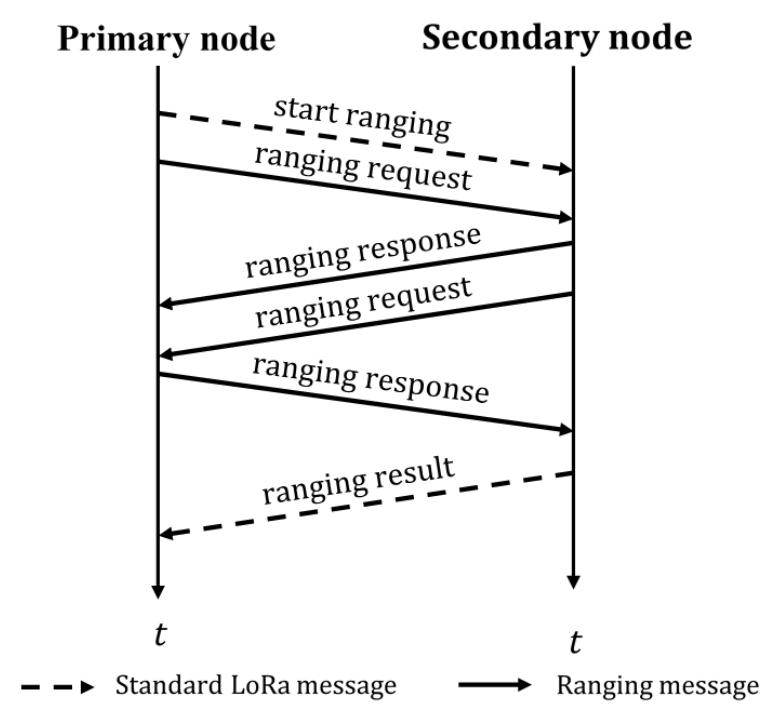

Fig. 2. Implemented back-to-back ranging with LoRa modulation

the relative oscillator offset can be eliminated by averaging the ranging distances obtained by the two individual measurements, assuming that there are no clock drifts during the measurement duration consisting of the back-to-back ranging procedure.

We term the node starting the back-to-back ranging primary node and the other node secondary node. Additional management messaging using standard LoRa messages are introduced for starting the back-to-back ranging procedure by the primary node and for exchanging the result of the second two-way ranging procedure between secondary and primary node.

For the measurements we developed our own hardware platform based on the Semtech SX1280 transceiver and an STM32 processor. The hardware includes a GPS receiver module for determining the ground truth position and an SDcard for storing measurements used for subsequent offline processing and analysis.

\section{Sources of Error}

Apart from the already considered relative oscillator offset error in (2), additional sources of error are discussed next. The temporal resolution capability of multipath components will limit the accuracy of the measured ranging distance in a multipath environment, since the best estimate for a ranging distance is the direct LOS path length. The multipath resolution capability, expressed as the difference in length of individual paths is according to section 2.1.3 in [8] approximately

$$
d_{R} \approx \frac{c}{B W}
$$

Considering the available bandwidths with the SX1280 transceiver this results in the multipath resolutions listed in TABLE I. The bandwidth $B W=125 \mathrm{kHz}$ is typically used with LoRa in the $868 \mathrm{MHz}$ frequency band and is included for comparison with the wider bandwidths available in the $2.4 \mathrm{GHz}$ ISM frequency band. Since in the best-case multipath components require at least $185 \mathrm{~m}$ difference in length to be fully separable, we expect this limitation to dominate the overall ranging distance error. 
TABLE I. THEORETICAL MULTIPATH RESOLUTION

\begin{tabular}{|c|c|}
\hline $\begin{array}{c}\text { Bandwidth BW } \\
{[\mathbf{k H z}]}\end{array}$ & $\begin{array}{c}\text { Multipath Resolution } \boldsymbol{d}_{\boldsymbol{R}} \\
{[\mathbf{m}]}\end{array}$ \\
\hline $125^{\mathrm{a}}$ & 2400 \\
\hline 406 & 739 \\
\hline 812 & 369 \\
\hline 1625 & 185 \\
\hline \multicolumn{2}{|c}{${ }^{\mathrm{a}}$. not supported by SX1280 } \\
\hline
\end{tabular}

The multipath resolution was tested in the lab with two LoRa nodes performing ranging measurements over a channel simulator (Elektrobit Propsim F32) with two configured paths. The LOS path measured $1800 \mathrm{~m}$ and the second path was $750 \mathrm{~m}$ longer and had $3 \mathrm{~dB}$ less power. This difference in path length is close to the theoretical multipath resolution for the LoRa bandwidth of $406 \mathrm{kHz}$ in TABLE I. The measured ranging distances for the different bandwidths listed in TABLE II. show that the smallest bandwidth of $406 \mathrm{kHz}$ is indeed not able to fully separate the paths and obtains a ranging distance of $1900 \mathrm{~m}$, while the larger bandwidths obtain values much closer to the true LOS path distance of $1800 \mathrm{~m}$ being simulated.

TABLE II. MEASURED MULTIPATH RESOLUTION

\begin{tabular}{|c|c|c|c|c|}
\hline \multirow{2}{*}{$\begin{array}{c}\text { Path 1 } \\
{[\mathbf{m}]}\end{array}$} & \multirow{2}{*}{$\begin{array}{c}\text { Path 2 } \\
{[\mathbf{m}]}\end{array}$} & \multicolumn{3}{|c|}{ Measured ranging distance [m] } \\
\cline { 3 - 5 } & $\mathbf{1 6 2 5} \mathbf{~ k H z}$ & $\mathbf{8 1 2} \mathbf{~ k H z}$ & $\mathbf{4 0 6} \mathbf{~ k H z}$ \\
\hline 1800 & 2550 & 1820 & 1820 & 1900 \\
\hline
\end{tabular}

Another source of error are additional delays in the implementation and the hardware (e.g. filters), where the hardware delays are frequency and bandwidth dependent. These can be determined and corrected using a calibration procedure described in section $\mathrm{D}$.

Finally, noise in the received signal causes ranging distance errors. The resulting theoretical lower bound of the standard deviation of the ranging distance $\sigma_{d}$ is given by the Cramér-Rao Lower Bound (CRLB) in section 6.1 in [9], which, after division by $\sqrt{2}$ to account for the averaging of the two two-way ranging measurements used in back-to-back ranging, is

$$
\sigma_{d}=c \cdot\left(\frac{1}{16 \cdot \pi^{2} \cdot S N R \cdot \sqrt{N} \cdot B W^{2} \cdot 2^{S F}}\right)^{1 / 2}
$$

with $N$ the configurable number of CSS symbols used in the ranging data. Based on (4) the largest standard deviation $\sigma_{d}=$ $1.26 \mathrm{~m}$ is obtained with $B W=403 \mathrm{kHz}$ and $S F=5$, assuming a Signal to Noise Ratio (SNR) of $S N R=12.5 \mathrm{~dB}$ and $N=15$. Thus, the expected error due to noise is far smaller than the limited multipath resolution in TABLE I.

\section{Calibration}

To remove fixed additional delays in the implementation, the system was calibrated using RF transmission over a coaxial cable of $75 \mathrm{~m}$ electrical length with one node placed in an RF shielding box to avoid coupling over the air between the two nodes. With this setup the offset-values for all $B W$ and $S F$ combinations were measured at $1 \mathrm{MHz}$ frequency steps with 100 measurements per parameter combination. These offset-values, in the range of several hundred meters, were then stored for correction of the ranging measurements. While performing calibration measurements the standard deviation was determined as $\sigma_{d}=2.11 \mathrm{~m}$ for $B W=403 \mathrm{kHz}, S F=5$, $S N R=12.5 \mathrm{~dB}$ and $N=15$, which is $67 \%$ higher than the theoretical lower bound given by (4).

Calibration can also remove the relative oscillator offsets between the two nodes in (2), but only if they do not change over time, e.g. due to temperature variations.

\section{RANGING MEASUREMENTS}

Ranging measurements were conducted with our SX1280 based hardware between two calibrated nodes in a flat, rural environment. The primary node had a fixed location with antenna height of $1.5 \mathrm{~m}$ above ground, while the secondary node was carried by a person and was mounted on a short mast with an antenna height of $2 \mathrm{~m}$ above ground to avoid obstruction of the antenna by the person. Both antennas were omni-directional. The secondary node covered a route of 8.2 $\mathrm{km}$ length at walking speed with distances of up to $1400 \mathrm{~m}$ between the two nodes, using the LoRa parameters $B W=$ $400 \mathrm{kHz}$ and $S F=8$ and performing around 25000 ranging measurements on the whole route. The smallest bandwidth was used to obtain a robust link-budget while $S F=8$ resulted in slightly lower standard deviations $\sigma_{d}$ than higher values of $S F$ during calibration measurements in the laboratory for $B W=400 \mathrm{kHz}$. The ground truth distance was determined from logged GPS coordinates.

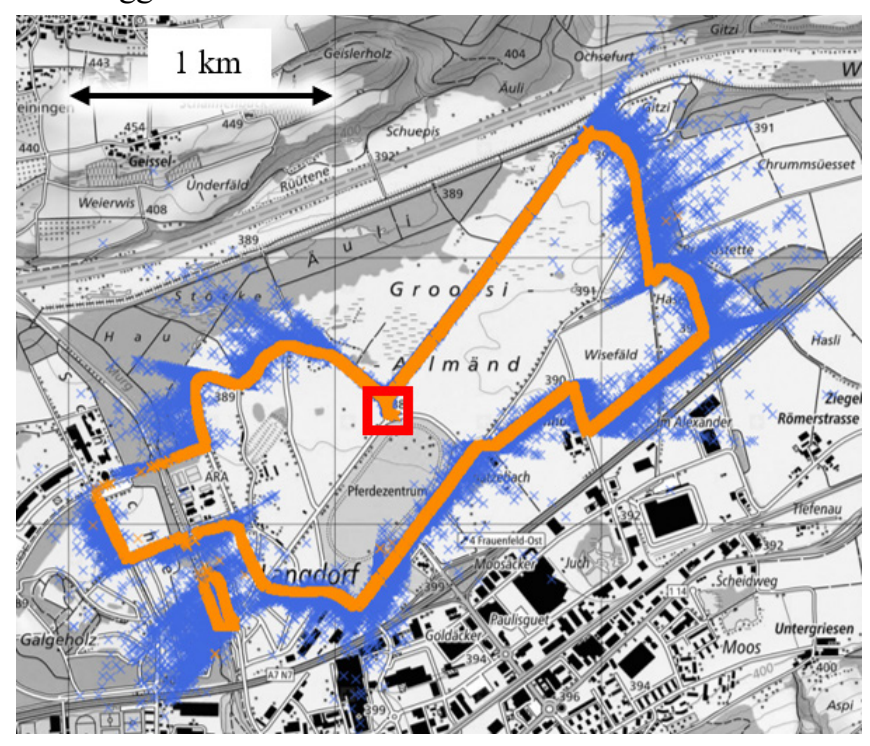

Fig. 3. Ranging test with ground truth route (orange crosses) and estimated ranging distances (blue crosses) marked on a radially extending line between the position of the fixed node (red square) and the ground truth position. Map: swisstopo

Fig. 3 shows the estimated ranging distances marked with blue crosses on a radially extending line between the position of the fixed node at the red square and the ground truth position of the mobile node. Compared to the ground truth route marked with orange crosses, it can be observed that in general the measured ranging distance over-estimates the true distance.

As can be observed from Fig. 4, the standard deviation of the distance error tends to increase with larger distances. We found that the increase in standard deviation correlates well with lower SNRs, as shown in Fig. 5, which shows the standard deviation of the distance error per SNR bin. A reduction in SNR is generally expected as the distance increases and the increase in standard deviation is therefore in 
line with the CRLB expression in (4). The outlier at $250 \mathrm{~m}$ distance was caused by a tree alley in that area, which resulted in shadowing and therefore a more multipath-dominated propagation. The standard deviation of the distance error over the whole route was $83.6 \mathrm{~m}$.

The multipath propagation in the test area and the limited multipath resolution are thought to have led to the observed

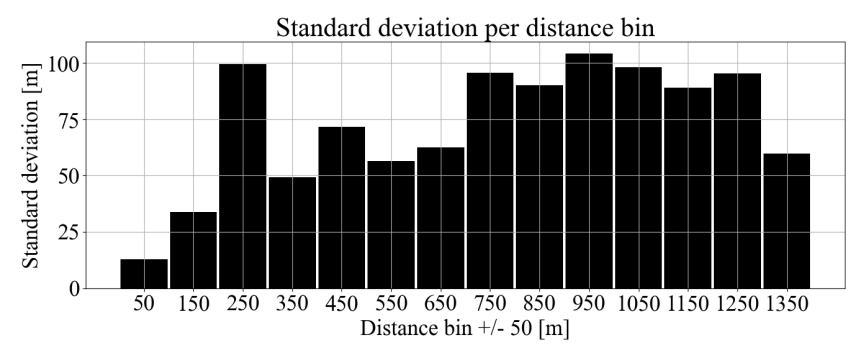

Fig. 4. Measured standard deviation of the ranging distance error per distance bin

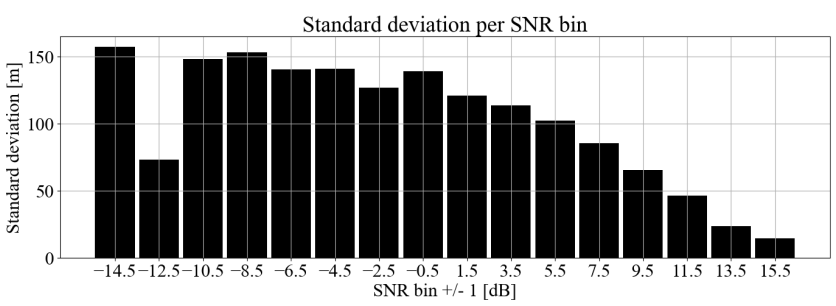

Fig. 5. Measured standard deviation of the ranging distance error per SNR bin

over-estimation of the ranging distance (i.e. positive mean distance error) for all distance bins, as shown in Fig. 6. The mean distance error tends to increase with larger distances, which could be explained with the LOS path becoming more blocked by obstacles as the distance increases. Again, an outlier at $250 \mathrm{~m}$ distance can be observed, which corresponds to the above-mentioned tree alley with increased multipath propagation. The maximum mean distance error was $91 \mathrm{~m}$ at $950 \mathrm{~m}$ distance in an area of low received signal strength due to shadowing by a forest, corresponding to a relative error of $9.6 \%$. The mean distance error over the whole route was $46.4 \mathrm{~m}$.

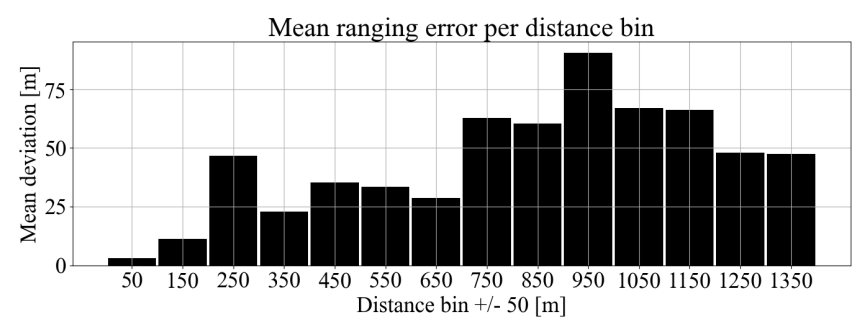

Fig. 6. Measured mean ranging distance error per distance bin

To reduce the mean ranging distance error, the following simple correction was derived using linear regression based on the measured ranging data. The corrected ranging distance $d_{c}$, given a measured ranging distance $20 \mathrm{~m} \leq d \leq 1400 \mathrm{~m}$, is

$$
d_{c}=0.95 \cdot d+1 \text {. }
$$

This correction extends the one proposed in section 8 in [9], which is valid for ranging distances $d<20 \mathrm{~m}$. By applying
(5) to the measured ranging distances, the mean distance error over the whole route could be reduced to $<10 \mathrm{~m}$.

Assuming that most practical applications will operate in a multipath environment and thus overestimate ranging distances, using a correction which is adapted to the specific environment is expected to be beneficial in most cases.

Further measurements were carried out in a suburban environment on a route of $2.5 \mathrm{~km}$ length and with distances of up to $500 \mathrm{~m}$ between the two nodes using the same LoRa parameter configuration. The resulting standard deviation of the distance error was $45 \mathrm{~m}$ and a mean distance error was $39 \mathrm{~m}$ over the whole route. The mean distance error was almost the same as in the rural environment, where the maximum distance between the two nodes were almost three times larger. This worse performance in the suburban environment may be attributed to the more multipathdominated propagation.

\section{Positioning BASED ON RANGING}

Based on LoRa ranging, we implemented a positioning method for coordinates in two dimensions. Using $K$ fixed reference nodes with the known coordinates of node $i$ being $\left(x_{i}, y_{i}\right)$, the unknown position of a mobile node with coordinates $(x, y)$ is first estimated as the least-squares solution to the set of equations for all ranging distances $d_{i}$ between the reference nodes $i=1,2, \ldots, K$ and the mobile node

$$
\begin{gathered}
\left(x_{1}-x\right)^{2}+\left(y_{1}-y\right)^{2}=d_{1}^{2} \\
\left(x_{2}-x\right)^{2}+\left(y_{2}-y\right)^{2}=d_{2}^{2} \\
\vdots \\
\left(x_{K}-x\right)^{2}+\left(y_{K}-y\right)^{2}=d_{K}^{2}
\end{gathered}
$$

After some manipulation of (6) the unknown coordinates $\boldsymbol{p}=\left[\begin{array}{ll}x & y\end{array}\right]^{T}$ can be obtained as the least-squares solution of this true-range multilateration problem as in equation (6.40) in [8]

$$
\boldsymbol{p}=\left(\boldsymbol{A}^{T} \cdot \boldsymbol{A}\right)^{-1} \cdot \boldsymbol{A}^{T} \cdot \boldsymbol{b}
$$

with

$$
\boldsymbol{A}=2 \cdot\left[\begin{array}{cc}
x_{2}-x_{1} & y_{2}-y_{1} \\
x_{3}-x_{2} & y_{3}-y_{2} \\
\vdots & \vdots \\
x_{K}-x_{K-1} & y_{K}-y_{K-1}
\end{array}\right]
$$

and

$$
\boldsymbol{b}=\left[\begin{array}{c}
-x_{1}^{2}+x_{2}^{2}-y_{1}^{2}+y_{2}^{2}+d_{1}^{2}-d_{2}^{2} \\
-x_{2}^{2}+x_{3}^{2}-y_{2}^{2}+y_{3}^{2}+d_{2}^{2}-d_{3}^{2} \\
\vdots \\
-x_{K-1}^{2}+x_{K}^{2}-y_{K-1}^{2}+y_{K}^{2}+d_{K-1}^{2}-d_{K}^{2}
\end{array}\right] .
$$

These position estimates are then filtered using a timediscrete, linear Kalman filter [10] for tracking of the mobile node with the state vector $\boldsymbol{x}_{n}$ at time $n$ comprised of position,

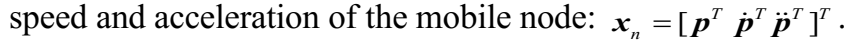


The tracking position prediction is

$$
\hat{\boldsymbol{p}}_{n}=\boldsymbol{p}_{n-1}+\dot{\boldsymbol{p}}_{n-1} \cdot \Delta t+\frac{1}{2} \cdot \ddot{\boldsymbol{p}}_{n-1} \cdot \Delta t^{2}
$$

with initialization $\boldsymbol{x}_{0}=\left[\begin{array}{lllll}\boldsymbol{p}_{0}^{T} & 0 & 0 & 0 & 0\end{array}\right]^{T}$, where $\boldsymbol{p}_{0}=\left[\begin{array}{lll}x_{\text {true }} & y_{\text {true }}\end{array}\right]^{T}$ are the true coordinates of the starting position of the mobile node. The Kalman filter only uses the positions obtained from LoRa ranging with (7) as measurement inputs. The state transition matrix can be obtained for $\Delta t=1 \mathrm{~s}$ as

$$
\boldsymbol{A}=\left[\begin{array}{cccccc}
1 & 0 & 1 & 0 & 0.5 & 0 \\
0 & 1 & 0 & 1 & 0 & 0.5 \\
0 & 0 & 1 & 0 & 1 & 0 \\
0 & 0 & 0 & 1 & 0 & 1 \\
0 & 0 & 0 & 0 & 1 & 0 \\
0 & 0 & 0 & 0 & 0 & 1
\end{array}\right],
$$

the observation model as

$$
\boldsymbol{C}=\left[\begin{array}{llllll}
1 & 0 & 0 & 0 & 0 & 0 \\
0 & 1 & 0 & 0 & 0 & 0
\end{array}\right],
$$

and the covariance matrix of the observation noise, whose values are based on the observed variance of the estimated positions, as

$$
\boldsymbol{Q}_{v}=\left[\begin{array}{cc}
1000 & 0 \\
0 & 1000
\end{array}\right] \text {. }
$$

\section{POSITIONING MEASUREMENTS}

The positioning method and tracking was evaluated with outdoor tests in a flat, rural environment using $K=4$ fixed reference nodes located in the edges of a quadrangular area measuring about $400 \mathrm{~m} \times 130 \mathrm{~m}$. The LoRa parameters were $B W=400 \mathrm{kHz}$ and $S F=8$. All ranging distances $d_{1}, d_{2}, \ldots, d_{K}$ and the estimated position $\boldsymbol{p}_{\mathrm{n}}$ of the mobile node are updated once per second, with $\Delta t=1 \mathrm{~s}$. Omni-directional antennas at a height of $1.5 \mathrm{~m}$ for the reference nodes and $2 \mathrm{~m}$ for the handheld mobile node were used with our SX1280 based hardware. To increase the accuracy of the estimated position, the measured ranging distances were first corrected using (5).

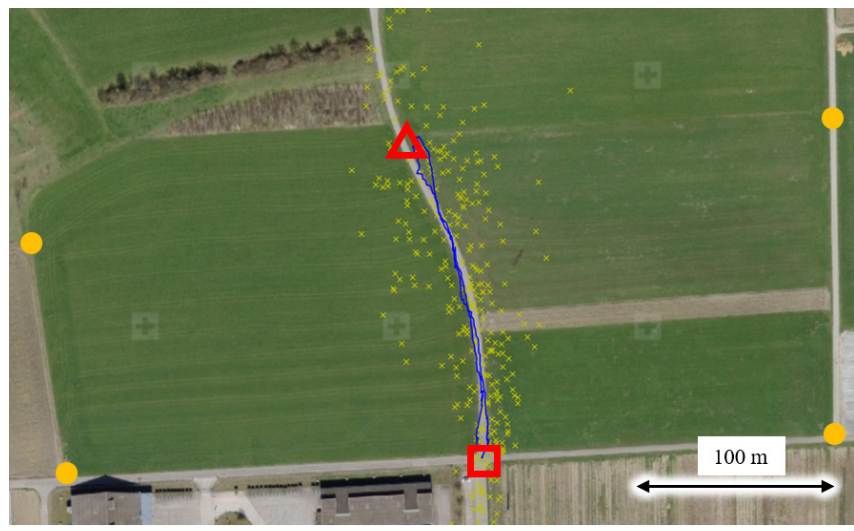

Fig. 7. Positioning Test with estimated positions using LoRa ranging directly (yellow crosses) and tracking with Kalman filtering (blue line). The orange circles indicate the reference nodes. Aerial view: swisstopo
Fig. 7 shows the test area with the 4 reference nodes marked with orange circles, the estimated positions of the mobile node directly obtained from LoRa ranging in (7) marked with yellow crosses and the estimated tracking using the additional Kalman filter in (10) marked with an interpolated blue line. The true route of the mobile node carried by a person started at the red square, followed the path to the red triangle and returned to the red square. Based on the geometry of the setup, the highest Dilution of Precision (DOP) can be expected at the start and return-points.

Positioning using the LoRa ranging measurements directly according to (7) resulted in a Distance Root Mean Square (DRMS) error of around $30 \mathrm{~m}$, while the use of the Kalman filter in (10) for tracking reduced the DRMS error to around $5 \mathrm{~m}$.

It should be noted that these measurements were performed in an environment with a dominant LOS path, the accuracy is expected to be worse in an environment with obstacles between the nodes. Improvements would be possible by using an Inertial Measurement Unit (IMU) sensor additionally to the LoRa ranging measurements to estimate the position of a mobile node.

\section{CONCLUSIONS}

As expected, the LoRa ranging measurements in the 2.4 $\mathrm{GHz}$ ISM frequency band were impacted by the limited multipath resolution capability, resulting in an overestimation of the ranging distances. This was observed over all distances in the outdoor measurements performed in a rural and a suburban environment. The variance and the mean of the ranging distance error both increased with higher distances due to a reduction in SNR and a likely increase in multipath propagation. Despite this, with a measured mean distance error of $46.4 \mathrm{~m}$ over true distances of up to $1400 \mathrm{~m}$ in a rural environment, the LoRa modulation is considered suitable for determining approximate distances. Further improvements are possible using corrections specific for the propagation environment to reduce the over-estimation of the ranging distance.

Positioning based on LoRa ranging obtained a DRMS of around $30 \mathrm{~m}$ for a mobile node in a rural outdoor area measuring about $400 \mathrm{~m} \times 130 \mathrm{~m}$, which allows approximate positioning without the use of GNSS. Tracking of the mobile node using Kalman filtering reduced the DRMS to around $5 \mathrm{~m}$. Further improvements would be possible for a mobile node by combining the estimated position with measurements from an IMU sensor. Compared to GNSS, positioning with LoRa however relies on the deployment of additional infrastructure in the form of reference nodes.

While the measurements were performed with the Semtech SX1280 transceiver with built-in ranging engine, the trends and conclusions are also applicable to other LoRa devices operating in the $2.4 \mathrm{GHz}$ band using comparable bandwidths.

\section{REFERENCES}

[1] J. P. Shanmuga Sundaram, W. Du and Z. Zhao, "A Survey on LoRa Networking: Research Problems, Current Solutions, and Open Issues," in IEEE Communications Surveys \& Tutorials, vol. 22, no. 1, pp. 371388, Firstquarter 2020.

[2] D. Plets, N. Podevijn, J. Trogh, L. Martens and W. Joseph, "Experimental Performance Evaluation of Outdoor TDoA and RSS 
Positioning in a Public LoRa Network," 2018 International Conference on Indoor Positioning and Indoor Navigation (IPIN), 2018, pp. 1-8.

[3] Bluetooth Core Version 5.2, Bluetooth SIG, 2019, Vol 6, Part B, p. 2869, Accessed: Apr. 26, 2021. [Online]. Available: https:/www.bluetooth.com/specifications/specs/

[4] F. Wolf, K. Le Déroff, S. de Rivaz, N. Deparis, F. Dehmas and J. Cances, "Benchmarking of Narrowband LPWA Physical Layer Ranging Technologies," 2019 16th Workshop on Positioning, Navigation and Communications (WPNC), Bremen, Germany, 2019, pp. 1-6.

[5] F. R. Andersen, K. D. Ballal, M. N. Petersen and S. Ruepp, "Ranging Capabilities of LoRa $2.4 \mathrm{GHz}$," 2020 IEEE 6th World Forum on Internet of Things (WF-IoT), New Orleans, LA, USA, 2020, pp. 1-5.

[6] Semtech Corporation, "LoRa ${ }^{\circledR}$ and LoRaWAN®: A Technical Overview," Accessed: Feb. 02, 2021. [Online]. Available: https://lora-
developers.semtech.com/uploads/documents/files/LoRa_and_LoRaW AN-A Tech Overview-Downloadable.pdf

[7] Semtech Corporation, "SX1280/SX1281/SX1282, Long Range, Low Power, 2.4 GHz Transceiver with Ranging Capability," Accessed: Feb. 02, 2021. [Online]. Available: https://www.semtech.com/products/ wireless-rf/24-ghz-transceivers/sx1280

[8] A. Bensky, Wireless Positioning Technologies and Applications. USA: Artech House, $2^{\text {nd }}$ ed., 2016.

[9] Semtech Corporation, "Application Note: An Introduction to Ranging with the SX1280 Transceiver," Accessed: Feb. 02, 2021. [Online]. Available: https://www.semtech.com/products/wireless-rf/24-ghztransceivers/sx 1280

[10] M. Hayes, Statistical Digital Signal Processing and Modelling. USA: John Wiley \& Sons, 1996, sec. 7.4. 